\title{
Références bibliographiques du dossier « les attentes éducatives des familles »
}

Hélène Beaucher

\section{OpenEdition}

1 Journals

Édition électronique

URL : https://journals.openedition.org/ries/3151

DOI : 10.4000/ries.3151

ISSN : 2261-4265

Éditeur

France Education international

\section{Édition imprimée}

Date de publication : 1 avril 2013

Pagination : 135-146

ISBN : 978-2-85420-599-2

ISSN : 1254-4590

Référence électronique

Hélène Beaucher, "Références bibliographiques du dossier " les attentes éducatives des familles » », Revue internationale d'éducation de Sèvres [En ligne], 62 I avril 2013, mis en ligne le 01 avril 2013, consulté le 05 juillet 2021. URL : http://journals.openedition.org/ries/3151 ; DOI : https://doi.org/ 10.4000/ries.3151 


\section{Références \\ bibliographiques}

\section{Hélène Beaucher}

La scolarité a pris une place qu'elle n'avait jamais occupée auparavant dans les familles. Depuis une quarantaine d'années, celles-ci ont connu des évolutions importantes, qu'il s'agisse de la structure familiale elle-même ou du rôle et des responsabilités des parents. Ces changements ont entraîné de nouvelles attentes des parents en matière d'éducation de leurs enfants.

Cette bibliographie non exhaustive est organisée en six parties. Le choix s'est porté sur des publications récentes. Des références liminaires apportent quelques éléments de cadrage. La deuxième partie recense des publications qui illustrent l'importance et la diversité des attentes éducatives des familles. La troisième partie est axée sur les différences sociales et ethniques observables dans le rapport à l'école, les aspirations et les pratiques éducatives. La partie suivante porte sur quelques pratiques éducatives et stratégies parentales, notamment le choix de l'école, l'accompagnement à la scolarité et l'implication dans les établissements scolaires. Les effets de l'implication familiale en termes de résultats scolaires sont examinés dans la cinquième partie. Enfin, quelques références portant sur l'éducation familiale - une forme d'intervention qui repose sur le développement des compétences parentales - complètent cette bibliographie.

Bibliographie arrêtée le 15 février 2013.

\section{ÉLÉMENTS DE CADRAGE}

\section{BLAIS Marie-Claude, GAUCHET Marcel, OTTAVI Dominique, Conditions de} l'éducation, Stock/Paris, 2008, 265 p. (Les essais)

Les auteurs proposent une réflexion sur ce qui fait obstacle aujourd'hui à la transmission éducative. Toute une série de conditions, qui servaient de socle à l'institution scolaire, ont été modifiées. Ces évolutions récentes poussent les familles à se décharger de leur ancien rôle de socialisation sur l'école, tout en contestant celle-ci et ses règles institutionnelles au nom de leurs propres valeurs affectives. L'analyse se développe sur quatre points : les relations de l'école avec la famille, le sens des savoirs qu'elle dispense, l'autorité dont elle a besoin et sa place dans la société.

BRINBAUM Yaël, "Attentes éducatives des familles ", in Dictionnaire de l'éducation, VAN ZANTEN Agnès (sous la direction de), Presses universitaires de Francel Paris, 2008, p. 24-26

Les attentes éducatives des familles ont évolué fortement depuis les années 1960, en lien avec l'allongement de la scolarisation, les modifications dans la famille, la place accordée à l'école au sein des familles, l'évolution du marché du travail et le chômage des jeunes. Si la forte élévation des attentes éducatives est commune à tous les milieux sociaux, y compris parmi les familles immigrées, la préoccupation scolaire se traduit par des formes diversifiées de mobilisation. 
DE SINGLY François, Sociologie de la famille contemporaine : domaines et approches, Armand Colin/Paris, 2010, 128 p. (4e édition)

Depuis la fin du XIX ${ }^{\mathrm{e}}$ siècle, la famille s'est transformée. Jusqu'aux années 1960, se sont imposés l'évidence du mariage amoureux, le repli sur le bonheur familial, et le soutien de l'État-Providence. Sont apparus par la suite l'instabilité conjugale, le pluralisme des formes familiales, la montée des normes psychologiques et la force du processus d'individualisation. En conséquence, la famille, prise entre les exigences de la vie commune et celle des individus, est soumise à de fortes tensions.

DUBET François (sous la direction de), École, familles : le malentendu, Textuel/ Paris, 1997, 170 p. (Le penser vivre)

Les querelles entre les familles et l'école ne cessent d'alimenter les remises en cause de l'institution scolaire. D'où vient ce malentendu ? L'école républicaine est devenue un mythe. La massification scolaire, la discrimination sociale ont changé les règles du jeu. Les familles sont désormais inégales face à un système scolaire transformé en "marché » au détriment des classes populaires. Face aux nouvelles attentes et attitudes des parents et des élèves, de nouveaux contrats parents enseignants sont à réinventer.

\section{LAHIRE Bernard, Tableaux de familles : heurs et malheurs scolaires en milieux populaires, Gallimard, Éditions du Seuil/Paris, 1995, 297 p.}

La scolarité des enfants de milieux populaires connaît, en raison de différences internes à ces milieux, des variations considérables. Il existe, selon les situations, des phénomènes de dissonances et de consonances entre des configurations familiales populaires et l'univers scolaire. L'auteur cherche à expliquer quelles sont les conditions de transmission du capital culturel familial et comment, en l'absence d'une telle transmission, les enfants peuvent cependant s'approprier les savoirs scolaires.

\section{Des attentes Élevées ET DIVERSES}

BOETHEL Martha, Diversity, School, Family and Community Connections: Annual Synthesis 2003, National Center for Family and Community Connections with Schools/Austin, 2003, 100 p. disponibles sur: http://www.sedl.org [en ligne]

Cette étude montre que quelle que soit l'appartenance ethnique, culturelle ou sociale, la plupart des familles ont pour leurs enfants des aspirations scolaires élevées. Si les familles appartenant à des minorités ethniques et culturelles sont activement impliquées dans la scolarisation de leurs enfants, leurs types de participation peuvent toutefois différer de ceux des familles blanches anglo-saxonnes protestantes. Les facteurs socioéconomiques influencent également les types de participation parmi les familles à faible revenu.

BRIDGELAND John M., DILULIO John J., STREETER Ryan T, et al., One Dream, Two Realities: Perspectives of Parents on America's High School, Civic Enterprises/ Washington, 2008, 39 p. disponibles sur : http://www.hartresearch.com [en ligne] Comment les parents perçoivent-ils l'école aux États-Unis? Quelles sont leurs attentes éducatives ? S'appuyant sur les résultats d'une enquête menée auprès de parents d'élèves, ce rapport révèle que tous les parents ont des aspirations élevées, et qu'ils considèrent que leur implication est essentielle pour la réussite de leurs enfants. Les parents de milieux défavorisés éprouvent néanmoins des difficultés à accompagner leur perception positive par des actions concrètes, par manque de temps et parce qu'ils perçoivent une distance entre eux et l'école. 
CERI : Centre pour la recherche et l'innovation dans l'enseignement, L'école face aux attentes du public : faits et enjeux, OCDE/Paris, 2006, 163 p. (L'école de demain)

Cet ouvrage examine et clarifie les différents aspects du concept de demande en éducation. À partir de données internationales provenant de onze pays de l'OCDE, il met en lumière les attitudes et les attentes du public. L'éducation occupe une place élevée dans les priorités du public. Lorsque l'on demande aux parents ce qu'il est le plus important d'atteindre à travers l'école, deux grands groupes se dégagent. Dans le premier (aspect scolaire/instrumental), l'école doit inculquer les connaissances et compétences de base, afin que les enfants aient de bons résultats scolaires et de bonnes opportunités d'emploi ; dans le second (aspect humaniste), l'école doit veiller au bienêtre général des enfants et leur apprendre à développer des qualités humaines.

\section{CROZIER Gill, Parents, Children and the School Experience: Asian families'} perspectives: research report, Economic and Social Research/Swindon, 2004, 29 p. disponibles sur: http://www.esrc.ac.uk [en ligne]

Ce rapport présente les résultats d'une enquête menée en Angleterre auprès de familles du Pakistan et du Bangladesh. Tous les parents, quelles que soient leurs caractéristiques ethniques et culturelles, attachent de l'importance à l'éducation et à la réussite scolaire de leurs enfants. En revanche, ils s'estiment très peu informés sur le fonctionnement de l'école et ont le sentiment que les enseignants n'attachent que peu d'importance à leur information.

\section{JACOB Brian A., LEFGREN Lars, "What Do Parents Value in Education? An} Empirical Investigation of Parents' Revealed Preference for Teachers ", The Quarterly Journal of Economics, 2007, vol. 122, $n^{\circ} 4$, p. 1603-1638

Quels types d'enseignants préferent les parents d'enfants scolarisés dans l'enseignement primaire? À la lumière des résultats d'une recherche menée aux États-Unis, les auteurs démontrent que les parents qui interviennent auprès d'un chef d'établissement, afin de choisir un enseignant, ne privilégient pas en premier lieu les enseignants décrits comme performants dans l'apprentissage de la lecture ou des mathématiques. Le bien-être de l'enfant prime, et les parents choisissent les enseignants décrits comme les plus populaires auprès des enfants.

"Les attentes des parents vis-à-vis de l'institution scolaire », Le Débat, 2004, vol. 5, $n^{\circ} 132$, p. 91-97

Cet article présente les conclusions d'une enquête sur les représentations de l'école idéale chez les parents et les enseignants en France, en particulier sur ce que sont les attentes des familles. Il fait ressortir le caractère consensuel de l'image qui se dégage : l'idée de «l'école sanctuaire » s'efface au profit d' «attentes unanimes d'ouverture de l'école à la société ». Cette image repose sur quatre présupposés : l'école doit réparer la société ; l'école a pour mission de faciliter la poursuite du bonheur de l'individu; l'individu est la base de la société ; l'opacité de l'école vis-à-vis des parents n'est plus légitime.

\section{PRÉVÔT Olivier, "Attentes des familles à l'égard de l'école : une enquête auprès}

de 2492 parents " in Construire une " communauté éducative " : un partenariat famille-école-association, PITHON Gérard, ASDIH Carole, LARIVEE Serge J. (sous la direction de), De Boeck/Bruxelles, 2008 (Perspectives en éducation et formation), p. 37-50

En s'appuyant sur une enquête quantitative, cet article tente de cerner les attentes en matière de soutien à la parentalité de parents du Territoire de Belfort (France). La scolarité des enfants, leur éducation, leur santé, leurs difficultés comportementales constituent les sources les plus importantes de leurs préoccupations quotidiennes. Les attentes de soutien des parents portent d'abord sur le système de communication entre les membres de la famille, puis sur la gestion des rôles des uns envers les autres, et notamment des conflits qui en découlent. Il s'agit, en quelque sorte, d'une quête pour une nouvelle forme de "gouvernance familiale ». 
TERRISSE Bernard, et al., "Les besoins d'information et de formation à l'exercice des responsabilités éducatives des parents québécois ", La revue internationale de l'éducation familiale, $2008, n^{\circ} 23$, p. 81-101

Cet article présente les résultats d'une recherche menée auprès de parents québécois sur leurs besoins d'information et de formation à l'exercice de leurs responsabilités éducatives. Les données montrent clairement que ceux-ci désirent davantage être formés qu'informés.

\title{
LE RAPPORT À L’ÉDUCATION : DIFFÉRENCES SOCIALES ET ETHNIQUES
}

\begin{abstract}
BRINBAUM Yaël, KIEFFER Annick, "D'une génération à l'autre, les aspirations éducatives des familles immigrées : ambition et persévérance ", Éducation et formations, septembre 2005, $n^{\circ}$ 72, p. 53-75

Les aspirations des parents immigrés, dont la nature differe selon l'origine nationale, apparaissent plus élevées que celles des Français d'origine issus du même milieu social. Cependant, des décalages apparaissent entre les aspirations des parents, celles de leurs enfants et les scolarités effectives de ces derniers. Ces décalages peuvent engendrer des frustrations chez certains parents et enfants.
\end{abstract}

DENIGER Marc-André, ABDOULAYE Anne., DUBÉ Sylvain., et al., Les représentations du système scolaire des familles issues de milieux défavorisés, Groupe de recherche sur l'éducation en milieu défavorisé/Montréal, juillet 2009, 28 p. disponibles sur: https://www.oirs.ulaval.ca [en ligne]

Les auteurs présentent les résultats d'une recension des écrits sur les représentations du système scolaire de familles de milieux défavorisés. Ces familles ont une représentation positive de l'école, perçue comme essentielle pour un meilleur avenir. Malgré l'importance qu'elles y accordent, leur manque d'emprise sur le système scolaire accentue leur sentiment de distance face à l'école.

GOMBERT Philippe, L'École et ses stratèges : les pratiques éducatives des nouvelles classes supérieures, Presses universitaires de Rennes/Rennes, 2008, 250 p.

Cet ouvrage explore les stratégies scolaires des fractions inférieures des classes supérieures, et montre quelles en sont les conséquences en termes de régulation des pratiques éducatives. Deux préoccupations centrales, sources de tensions entre deux principes de socialisation, caractérisent l'éducation familiale de ces classes supérieures : l'idéal d'autonomie et d'épanouissement individuel ; le culte de la performance et les stratégies scolaires de long terme. L'auteur montre que les valeurs de ces parents sont en adéquation avec le paradigme dominant qui s'est imposé, à partir des années 1980, autour des idées de pluralisme scolaire, de néo-libéralisme éducatif et de parents consommateurs.

GREMOIN Myriam, HUTTER Valérie, "Stratégies parentales et dynamisme éducatif : l'exemple de familles migrantes suisses ", in Construire une "communauté éducative »: un partenariat famille-école-association, PITHON Gérard, ASDIH Carole, LARIVEE Serge J. (sous la direction de), De Boeck/Bruxelles, 2008, p. 129-146 (Perspectives en éducation et formation)

Cette contribution rend compte des stratégies éducatives opérées, de manière volontaire, par les familles migrantes en Suisse. L'école revêt une importance capitale pour ces parents qui souhaitent que leurs enfants puissent avoir un quotidien plus facile que le leur. Ils s'engagent 
activement dans l'avenir de leurs enfants, leur demandent de s'investir dans leur réussite et leur font comprendre l'importance de l'école. Les auteures mettent en évidence l'effort constant que les personnes migrantes fournissent afin de comprendre, faciliter et faire évoluer une vie familiale dont les repères sont en mutation, suite à la scolarisation des enfants aînés.

\section{LAREAU Annette, Unequal Childhoods: Class, Race, and Family Life, University of California Press/Berkeley, 2011, 480 p. (2e édition)}

L'auteure revisite les thèmes développés dans la première édition de cet ouvrage, en réinterrogeant douze familles américaines étudiées en 2003. Elle s'intéresse à l'impact de la classe sociale sur la vie des familles et sur le devenir des enfants. En matière de style éducatif, les différences entre les classes sociales sont frappantes. Contrairement aux familles de la classe moyenne, les familles de milieu populaire manifestent un sentiment de défiance face à l'école. Plus que le facteur ethnique, c'est le facteur socio-économique qui influence les résultats scolaires et le devenir des enfants. Les résultats de cette deuxième étude confirment les conclusions de la première.

\section{LENOIR Annick, "La tension instruction-socialisation dans les relations école-} familles immigrantes : une question de reconnaissance?", in Les pratiques enseignantes entre instruire et socialiser : regards internationaux, LENOIR Yves, TURPIN Frédéric (Sous la direction de), Presses de l'Université de Laval, 2012, p. 395-422 (Formation et profession)

Cette contribution repose sur l'hypothèse, soutenue par des recherches menées au Canada, que les différents entre l'école et les familles issues de l'immigration découlent principalement de deux logiques différentes quant aux missions de l'école. L'auteure interroge la tension entre l'instruction et la socialisation pour relever les deux logiques distinctes et les différentes conduites qui en découlent. Si les enseignants privilégient la mission de socialisation, les familles issues de l'immigration conçoivent l'école comme le lieu devant être réservé à l'instruction, le processus de socialisation leur appartenant exclusivement.

PÉRIER Pierre, "Entre les parents et l'école ", in L'ordre scolaire négocié : parents, élèves, professeurs dans les quartiers difficiles, Presses universitaire de Rennes/Rennes, 2010, p. 56-93 (Le sens social)

Les espoirs placés dans l'école, la montée de la préoccupation et de l'investissement scolaires des parents, signalent l'importance que la scolarité des enfants a prise dans les familles. Les développements proposés cherchent à caractériser le type de rapport des familles populaires à l'école et les formes concrètes de leur investissement. S'appuyant sur des enquêtes auprès de parents, l'auteur s'intéresse à la manière dont les familles populaires s'approprient l'enjeu de la scolarité, composent avec les normes et exigences d'une école dont elles attendent et dépendent beaucoup tout à la fois.

THIN Daniel, "Un travail parental sous tension: les pratiques des familles populaires à l'épreuve des logiques scolaires ", Informations sociales, $n^{\circ} 154$, 2009, p. 70-76

S'appuyant sur plusieurs recherches concernant les relations entre les familles populaires et l'école, l'article s'intéresse aux pratiques des parents à l'égard de la scolarité de leurs enfants, mais également aux pratiques domestiques et à celles qui ont trait au mode d'autorité parental. L'auteur montre que les pratiques de ces familles sont sous-tendues par la tension entre les logiques socialisatrices des familles populaires et les logiques scolaires qui s'imposent à elles. 
VAN ZANTEN Agnès, "Le travail éducatif parental dans les classes moyennes et supérieures : deux modes contrastés d'encadrement des pratiques et des choix des enfants ", Informations sociales, 2009, vol. 4, $n^{\circ} 154$, p. 80-87

Le travail éducatif parental revêt des formes différentes dans les classes moyennes et supérieures d'un côté et dans les classes populaires de l'autre, en raison de l'inégale répartition des ressources, mais aussi de conceptions différentes de ce qui constitue un bon encadrement éducatif. Les membres du premier groupe mettent en œuvre un ensemble de pratiques cohérentes, qui leur permettent de contrôler la socialisation et la scolarisation de leurs enfants, à travers deux modalités : un encerclement protecteur autour de l'enfant visant à recréer un entre soi de classe, ou un modelage en douceur visant à reproduire des habitus intellectuels et sociaux.

\section{FOCUS SUR QUELQUES PRATIQUES ÉDUCATIVES}

\section{Choix d'une école}

BURGESS Simon, GREAVES Ellen, VIGNOLES Anna, et al., What Parents Want: School Preferences and School Choice, CMPO: Centre for Market and Public Organisation, University of Bristol/Bristol, 2009, 62 p. disponibles sur: http:// www.bristol.ac.uk/ [en ligne]

Ce document de travail s'intéresse aux différents facteurs qui déterminent le choix d'un établissement scolaire par les parents en Angleterre. L'analyse est fondée sur des enquêtes, des données socio-économiques et des informations sur les caractéristiques de l'école (performance scolaire, composition socio-économique et ethnique, caractère confessionnel de l'établissement). Les familles accordent une grande importance à la performance scolaire des écoles. Elles accordent également beaucoup d'importance à leur composition, préférant les écoles comportant de faibles fractions d'enfants issus de familles défavorisées.

DENHERE Chris, "Choosing a high school: Parental considerations ", Prime Research on Education, juillet 2012, vol. 2, $n^{\circ}$ 6, p. 289-293

Cette étude porte sur les choix des parents en matière d'établissement d'enseignement secondaire au Zimbabwe. Les auteurs ont soumis un questionnaire aux familles. Les résultats suggèrent que cinq principaux critères orientent le choix des parents : d'abord les résultats aux examens, puis le coût de la scolarité, la discipline, les équipements de l'établissement, et enfin le caractère confessionnel de ce dernier.

NISHIMURA Mikiko, YAMANO Takashi, School Choice between Public and Private Primary Schools under the Free Primary Education Policy in Rural Kenya, GRIPS Policy Information Center/Tokyo, 2008, 22 p. disponibles sur: http://www3.grips.ac [en ligne]

Depuis l'instauration de la gratuité de l'enseignement primaire en 2003 au Kenya, le nombre d'écoles privées n'a cessé d'augmenter. La proportion d'enfants fréquentant les écoles primaires privées a progressé de $11,5 \%$. Les auteurs suggèrent que les parents qui ont en les moyens réagissent à la dégradation de la qualité de l'enseignement public en transférant leurs enfants dans les écoles privées. 
ROLAND Nicolas, "Choisir une école dans les milieux populaires : du choix de l'école par les familles issues de milieux populaires ", Education \& Formation, juillet 2012, $n^{\circ}$ e-297, p. 75-96 disponible sur : http://ute3.umh.ac.be/revues [en ligne]

Cette étude tente de mettre au jour les stratégies qui sous-tendent les processus de choix d'un établissement scolaire secondaire en Communauté française de Belgique par les familles issues de milieux populaires. L'auteur soutient que l'idée d'un abandon et d'un non-choix dans les milieux populaires s'avère être un mythe. Les familles mettent en œuvre, dans leurs processus de choix d'un établissement scolaire, diverses stratégies - rationnelles, sociales ou sociorationnelles - influencées par les variables « diplôme » et « emploi ».

\section{VAN ZANTEN Agnès, Choisir son école: stratégies familiales et médiations} locales, Presses universitaires de France/Paris, (Le lien social), 2009, 283 p.

La plupart des parents souhaitent avoir le droit de choisir l'établissement de leur enfant. Certains optent pour le secteur privé, d'autres contournent la carte scolaire du secteur public. Un autre type de choix est celui de parents qui, envoyant leurs enfants dans les établissements du quartier, s'y investissent fortement pour surveiller leur scolarité. Prenant appui sur une enquête menée dans la périphérie parisienne, cet ouvrage s'intéresse aux parents des classes moyennes. Il étudie les clivages entre les différentes fractions de ce groupe social autour de choix qui constituent pour leurs membres d'importants enjeux, tant du point de vue des carrières scolaires de leurs enfants que de la protection de leur statut collectif. L'auteure analyse les différents déterminants du choix (visées, valeurs, raisons et ressources des parents), et examine aussi la façon dont ces choix se construisent dans des espaces en interaction avec l'offre éducative.

WASLANDER Sietske, PATER Cissy, VAN DER WEIDE Maartje, "Demand Side Mechanims: Parents and Choice ", in Markets in Education: An Analytical Review of Empirical Research on Market Mechanisms in Education, OECD/ Paris, OECD Education Working Papers, $n^{\circ}$ 52, 2010, p. 28-44 disponibles sur: http://www.oecd-ilibrary.org [en ligne]

$\mathrm{Au}$ cours des trois dernières décennies, de nombreux gouvernements ont introduit des mécanismes de marché au sein de leur système éducatif, valorisant le choix des parents d'élèves par des mesures telles que l'abolition des zones scolaires, la création de programmes accessibles à l'aide de chèques scolaires, ou la mise en place des écoles à charte. Cette contribution porte sur les critères qui déterminent les choix d'un établissement scolaire par les parents dans les pays de l'OCDE. Les facteurs les plus fréquemment pris en compte par les parents sont les performances scolaires, les caractéristiques du public de l'établissement, la distance entre l'école et le domicile, et le bien-être des enfants. Dans certaines zones urbaines, la question de la sécurité se révèle un critère de choix important.

\section{Pratiques d'accompagnement à la scolarité}

BRAY Mark, L'ombre du système éducatif : quel soutien scolaire privé, quelles politiques publiques?, IIEP-UNESCO/Paris, 2011, 140 p.

L'expression «l'ombre du système éducatif " (Shadow Education) fait référence au soutien scolaire privé, qui existe depuis longtemps dans certaines régions d'Asie de l'Est, et devient prééminent dans d'autres parties de l'Asie ainsi qu'en Afrique, en Amérique du nord et en Europe. L'auteur évalue l'ampleur, la nature et les implications du soutien scolaire privé dans différents et pays et régions du monde. Il identifie dans un second temps les éventuelles 
réponses que les gouvernements peuvent proposer pour déterminer les types de soutien scolaire souhaitables et mettre en place les politiques appropriées pour encadrer les plus problématiques d'entre eux. Il remarque que le recours au soutien scolaire est lié en grande partie au fait que les parents ne sont pas certains que les systèmes d'éducation répondent aux besoins de la société en général et de leurs enfants en particulier.

\section{KAKPO Séverine, Les devoirs à la maison : mobilisation et désorientation des} familles populaires, Presses Universitaires de France/Paris, 2012, 211 p. (Éducation et société)

L'auteure s'appuie sur une enquête ethnographique conduite auprès de familles populaires en France, pour mettre à jour les pratiques familiales d'accompagnement des devoirs et étudier les rapports qu'entretiennent logiques pédagogiques, familiales et logiques pédagogiques scolaires. Elle analyse la conduite des parents, totalement investis dans la production d'un travail qu'ils ne sont pas toujours en mesure d'accompagner, faute de ressources culturelles. Elle montre également qu'ils souffrent d'une désorientation pédagogique sur le plan des savoirs.

\section{OLLER Anne-Claudine, "Le coaching scolaire en France. Émergence d'un nouveau} marché éducatif ", Éducation comparée, 2011, vol. 6, p. 181-202

Cet article s'intéresse à l'émergence d'un nouveau marché scolaire et d'une nouvelle stratégie éducative en France : le coaching scolaire. La montée en puissance d'entreprises privées offrant un appui à la scolarité fait écho aux inquiétudes des parents, qui peuvent formuler par la suite une demande plus explicite d'accompagnement scolaire. Après avoir présenté le contenu de l'offre, l'auteure analyse, à partir d'une enquête, les raisons de recourir au coaching scolaire. Ce recours au coaching émane de parents aisés, essentiellement des cadres issus du monde de l'entreprise.

\section{Implication dans l’école}

\section{DALSHEIME-VAN DER TO Nadine, MURAT Francis, "Les parents et l'école en} France et en Europe ", Éducation et formations, décembre 2011, $n^{\circ}$ 80, p. 79-94

Les auteurs apportent un éclairage sur l'implication des parents dans le fonctionnement des établissements en France, qu'ils comparent, d'un point de vue plus descriptif et institutionnel, avec d'autres situations nationales. La participation des parents à la vie de l'établissement peut prendre des formes diverses : rencontre avec les professeurs, adhésion à une association de parents, d'élèves, accompagnement de sorties, etc. L'implication des parents varie fortement. Alors que certains s'inscrivent dans une association, d'autres n'ont presque jamais de contact avec l'établissement où leur enfant est scolarisé. Ces différences sont notamment liées au milieu social et aux résultats de l'enfant. En Europe, les parents tendent à avoir une place de plus en plus grande dans la vie des établissements.

OCDE, "Comment les parents peuvent-ils influer sur les structures d'éducation de leur enfant?", in Regards sur l'éducation 2010 : Panorama, OCDE/Paris, 2010, p. 464-473

Cet indicateur cherche à déterminer, dans les pays de l'OCDE, si les parents ont la possibilité d'influer sur l'établissement d'enseignement où leur enfant est scolarisé et à identifier les moyens qui s'offrent à eux pour le faire. Il analyse trois moyens d'expression officiels à la disposition des parents d'élèves : la participation à la gouvernance, la participation consultative, et le dépôt de plaintes ou de recours. Sur trente pays, dix-huit imposent que les parents d'élèves soient représentés au conseil de direction des établissements publics. 
RIZZI Felice, BRUNELLI Michele, FERDANDEZ Alfred, et al. (sous la direction de), L'implication parentale au sein de l'école : une approche innovante pour une éducation de qualité, L'Harmattan/Paris, 2011, 171 p.

Le projet IPPE (Indicateurs de Participation des Parents dans l'Enseignement obligatoire) avait pour but de construire des indicateurs afin de mesurer la participation des parents dans les systèmes éducatifs européens : droit d'information, droit de choisir l'école, droit de recours et droit de participation dans les organes formels. Après avoir explicité l'approche de l'éducation, basée sur les droits, les auteurs présentent les résultats obtenus pour chacun des quinze pays européens étudiés, qu'ils analysent dans une perspective comparatiste. Les auteurs insistent sur la nécessité de mettre en place au niveau européen des dispositifs reflétant les attentes et les opinions des parents, et de concevoir également de nouvelles formules de participation des parents.

WARZEE Alain, LE GOFF Françoise, LESAGE Gérard, et al., La place et le rôle des parents dans l'école : rapport à monsieur le ministre de l'Éducation nationale, de l'Enseignement supérieur et de la Recherche, Ministère de l'Éducation nationale, de l'Enseignement supérieur et de la Recherche/Paris, 2006, 87 p.

Ce rapport propose une réflexion sur le rôle et la place des parents à l'école en France. Les auteurs rappellent les principales dispositions juridiques en vigueur, et présentent un état des lieux complété par des recommandations. En France, le contrôle par les parents des programmes scolaires est limité. Les parents d'élèves souhaitent avoir un véritable statut qui leur reconnaisse des droits. Si leurs attentes en matière de réussite scolaire sont importantes - chaque famille attendant de l'école qu'elle favorise également ses projets particuliers -, les parents sont peu investis dans l'action collective. Une tension est souvent perceptible entre les parents et les enseignants. Ceux-ci considèrent parfois l'entrée des parents à l'école comme une intrusion, notamment dans le domaine pédagogique.

\section{IMPLICATION FAMILIALE ET RÉSULTATS SCOLAIRES}

BORGONOVI Francesca, MONTT Guillermo, Parental Involvement in Selected PISA Countries and Economies, OECD Education Working Papers, $n^{\circ} 73,2012$, 165 p. disponibles sur : http://www.oecd-ilibrary.org [en ligne]

En 2009, dans le cadre de l'enquête PISA, quatorze pays ont administré le questionnaire facultatif destiné aux parents. Ce document analyse le degré de l'engagement parental dans ces pays, ainsi que la relation entre cet engagement et certains processus cognitifs (performance en compréhension de l'écrit) et non cognitifs. Les résultats laissent penser que certaines formes d'engagement parental sont plus fortement corrélées que d'autres à ces processus. Si le degré d'engagement des parents varie entre les différents pays et, au sein de ces derniers, entre les différents sous-groupes de population, l'étude confirme que le renforcement de l'engagement parental peut améliorer les résultats des élèves.

DESLANDES Rollande, CLOUTIER Richard, "Pratiques parentales et réussite scolaire en fonction de la structure familiale et du genre des adolescents", Revue française de pédagogie, avril 2005, $n^{\circ} 15$, p. 161-174

Cet article porte sur la comparaison de certains indicateurs de la réussite scolaire et des pratiques éducatives des parents en fonction de la structure familiale et du genre des adolescents. Les pratiques éducatives sont étudiées sous l'angle des pratiques parentales dites de base (style parental) et des pratiques reliées au suivi scolaire. L'auteur a identifié les contextes familiaux susceptibles de fragiliser les adolescents en termes de réussite scolaire. 
EL NOKALI Nermeen E., BACHMAN Heather J., VOTRUBA-DRZAL Elisabeth, "Parent Involvement and Children's Academic and Social Development in Elementary School ", Child Development, 2010, vol. 81, $n^{\circ}$ 3, p. 988-1005

Cette étude, regroupant des données sur 1300 enfants, fait apparaître que l'engagement parental dès le préscolaire, par le soutien au progrès de l'enfant, la présence à l'école et les contacts rapprochés avec les professionnels, a des effets bénéfiques sur le bien-être et les compétences sociales de l'enfant, ce qui pourrait influencer positivement, à plus long terme, ses compétences académiques.

FEYFANT Annie, Les effets de l'éducation familiale sur la réussite scolaire, Dossier d'actualité de la Veille et Analyses, juin 2011, $n^{\circ}$ 63, 14 p. disponibles sur: http://ife.ens-lyon.fr/ife [en ligne]

Ce dossier s'intéresse à l'incidence de l'environnement familial sur la scolarisation des enfants. Les différences de style éducatif, les attitudes des familles par rapport à l'école, l'accompagnement à la scolarité par les parents et les différences sociales apportent des éléments de compréhension en lien avec la réussite ou l'échec scolaire.

GORARD Stephen, SEE Beng Huat, DAVIES Peter, The impact of attitudes and aspirations on educational attainment and participation, Joseph Rowntree Foundation/York, avril 2012, 103 p. disponibles sur : http://jrf.org.uk/ [en ligne]

Les attitudes, aspirations et comportements éducatifs des enfants et de leurs parents influentils réellement sur les résultats scolaires? Si, oui, quels sont les types de pratiques susceptibles d'améliorer ces résultats et de réduire les écarts entre niveaux d'éducation ? Si les auteurs n'ont pu établir de lien de cause à effet évident entre les aspirations et les résultats scolaires, ce rapport confirme, à l'aide de données quantitatives, que l'implication des parents dans l'éducation améliore les résultats scolaires de leurs enfants.

LE PAPE Marie-Clémence, VAN ZANTEN Agnès, "Les pratiques éducatives des familles ", in Sociologie du système éducatif. Les inégalités scolaires, DURUT-BELLAT Marie, VAN ZANTEN Agnès (sous la direction de), Presses universitaires de France/Paris, 2009 (Licence), p. 185-205

Ce chapitre constitue une synthèse des enjeux de la socialisation familiale, et en particulier les effets d'une certaine socialisation de classe. Dans un premier temps, les auteures montrent comment certaines valeurs et pratiques éducatives des parents contribuent à faciliter l'intégration de l'enfant au sein de l'univers scolaire. Puis elles analysent les stratégies des parents en détaillant les différents aspects de l'accompagnement familial de la scolarité.

OCDE, Let's read them a story!: the parent factor in education, OECD/Paris, 2012, $79 p$.

De nouveaux résultats provenant du programme PISA de l'OCDE montrent que l'implication des parents dans l'éducation est essentielle pour la réussite des enfants pendant leurs années scolaires et au-delà. Ce rapport examine la façon dont l'implication des parents influence les compétences de leurs enfants et le plaisir de la lecture. Il présente les formes d'activités les plus fortement associées à l'amélioration des performances en compréhension de l'écrit, et rend compte de programmes provenant du monde entier qui favorisent un engagement parental efficace. 
SCHNEIDER Barbara, KEESLER Venessa, MORLOCK Larissa, "Les influences familiales sur l'apprentissage et la socialisation des enfants " in Comment apprend-on? La recherche au service de la pratique, DUMONT Hanna, ISTANCE David, BENAVIDES Francisco (éd.), OCDE/Paris, 2010, p. 265-299

Les auteures examinent la nature de l'influence familiale sur l'apprentissage des enfants, et l'objet de cette influence. Les familles jouent un rôle décisif dans la formation des attentes éducatives et des aspirations professionnelles ainsi que dans la réussite scolaire. La socialisation reçue à la maison a une forte influence sur le développement de l'ambition et du sentiment d'efficacité personnelle. L'implication parentale dans la scolarité et les activités extra-scolaires a des résultats positifs, particulièrement lorsque ces dernières sont en cohérence avec les objectifs et les activités de l'école.

\section{VeRs Une COÉducation ?}

AVVISATI Francesco, GURGAND Marc, GUYON Nina, et al.; Quels effets attendre d'une politique d'implication des parents d'élèves dans les collèges? Les enseignements d'une expérimentation contrôlée : rapport pour le Haut Commissaire à la Jeunesse, 2010, École d'économie de Paris/Paris, 2010, 36 p.

Ce rapport évalue une expérimentation initiée en 2008 dans l'académie de Créteil. Le dispositif "La mallette des parents », trois rencontres-débats réunissant des parents d'élèves et des acteurs du collège, s'inscrivait dans une dynamique de co-éducation. Il devait permettre de consolider le lien entre le collège et des parents d'élèves volontaires, de pallier leurs difficultés de communication face à la complexité du système éducatif, et de favoriser un meilleur suivi scolaire de leurs enfants. L'évaluation fait ressortir un surcroît d'implication des parents volontaires, qui s'est traduit par une amélioration sensible du comportement des enfants, y compris chez les enfants de parents n'ayant pas participé au dispositif.

DOBBIE Will, FRYER Roland G., Are High-Quality Schools Enough to Close the Achievement Gap? Evidence from a Bold Social Experiment in Harlem, Harvard University/Harvard, 2009, 53 p. disponibles sur : http://www.economics.harvard.edu [en ligne]

Aux États-Unis, afin de venir en aide aux enfants défavorisés, le programme d'envergure Harlem Children's Zone (HCZ) propose aux parents et à leurs enfants des services allant de la naissance à la fin des études. Dans un centre, tous les services sont regroupés: une école (Charter school), des services de soutien à la parentalité, des services de soutien scolaire pour les enfants, des services de garde, une clinique, des activités sportives, etc. Le but de la recherche était d'évaluer les effets de ce programme. Il apparaît extrêmement efficace, augmentant considérablement les résultats scolaires des enfants.

HAMEL Marie-Pierre, LEMOINE Sylvain (sous la coordination de), Aider les parents à être parents : le soutien à la parentalité, une perspective internationale : rapport, Centre d'analyse stratégique/Paris, 2012, 258 p.

Dans l'ensemble des pays de l'OCDE, on assiste au développement de services de soutien à la parentalité. D'une part, les parents ressentent et expriment un besoin de soutien concernant l'éducation de leurs enfants, d'autre part, les pouvoirs publics s'emparent de la question avec un fort volontarisme depuis une vingtaine d'années. Alors que cette politique publique se structure progressivement en France, ce rapport éclaire la réflexion par une approche internationale, présentant plus de cinquante expériences et actions prometteuses. 
POURTOIS Jean-Pierre, BARRAS Christine, DESMET Huguette, et al., "Les programmes d'éducation parentale : un soutien à la parentalité ?" in Traité des sciences et des pratiques en éducation, BEILLEROT Jacky, MOSCONI Nicole (sous la direction de), Dunod/Paris, 2006, p. 171-185 (Psycho sup)

Cette contribution propose une généalogie et un cadrage théorique de la notion d'éducation parentale au niveau international, puis une typologie des programmes correspondants. Les programmes d'éducation parentale visent à ce que les parents améliorent leurs compétences éducatives, et en premier lieu, à leur faire acquérir une plus grande confiance en leurs capacités. Les auteurs soulignent que, tant dans le développement scolaire que dans les interventions auprès des familles, les programmes qui intègrent les parents sont efficaces, même à long terme.

SAINT-JACQUES Marie-Christine, TURCOTTE Daniel, OUBRAYRIE-ROUSSEL Nathalie (sous la direction de), "L'éducation familiale visant à soutenir les parents et les enfants vivant dans des contextes de risque : dossier ", Enfances, Familles, Générations, printemps 2012, $n^{\circ} 16$, p. 1-123

Ce dossier traite de l'éducation familiale, une forme d'intervention qui repose sur le principe que pour assurer le bien-être des enfants, il faut soutenir leurs parents en développant leurs compétences. Les articles font ressortir l'importance accordée au développement des compétences des parents et aux représentations des acteurs. L'examen de l'efficacité des programmes fait ressortir que la vulnérabilité des familles n'est pas uniquement liée à la précarité socioéconomique, mais aussi à l'évolution des formes familiales elles-mêmes. 